\title{
High-resolution operation of frequency-multiplexed transition-edge photon sensors
}

M. F. Cunningham ${ }^{\text {a) }}$, J. N. Ullom, T. Miyazaki, S. E. Labov

Lawrence Livermore National Laboratory, Livermore, CA 94551

John Clarke ${ }^{\text {b) }}$, T. M. Lanting, Adrian T. Lee ${ }^{\text {c) }}$, P. L. Richards ${ }^{\text {b) }}$, Jongsoo Yoon ${ }^{\text {d) }}$

Department of Physics, University of California, Berkeley, CA 94720

H. Spieler

Physics Division, Lawrence Berkeley National Laboratory, Berkeley, CA 94720

\begin{abstract}
:
We present the multiplexed readout of two gamma-ray microcalorimeters made from transition-edge sensors. We use a frequency-domain multiplexing scheme in which each sensor is biased at an identifying frequency. We show that the energy resolution of the sensors is unaffected by multiplexing, and that cross-talk between the sensors is negligible. Our results indicate the feasibility of multiplexing 30 sensors or more to one readout line.
\end{abstract}




\section{Footnotes:}

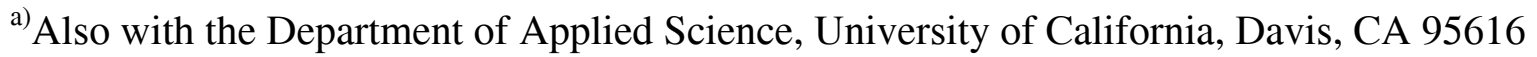

${ }^{b)}$ Also with the Materials Sciences Division, Lawrence Berkeley National Laboratory, Berkeley, CA 94720

${ }^{c}$ Also with the Physics Division, Lawrence Berkeley National Laboratory, Berkeley, CA 94720

d) present address: Department of Physics, University of Virginia, Charlottesville, VA 22903 


\section{Main Text:}

Transition-Edge Sensors (TESs) are a promising technology for precision measurements of electromagnetic radiation at gamma-ray, x-ray, optical, and far-infrared to millimeter wavelengths ${ }^{1,2,3,4}$. These sensors consist of superconducting thin films electrically biased in the resistive transition. Their sensitivity is a result of the strong dependence of resistance on temperature in the transition and the low specific heat and thermal conductivity of materials at typical operating temperatures near $100 \mathrm{mK}$. Because TES sensors can be reliably fabricated using well-established thin-film techniques, they are likely to be used in several important upcoming measurement programs which require imaging arrays of $10^{3}-10^{4}$ sensors. These programs include studies of the polarization of the cosmic microwave background, the planned X-ray satellites XEUS and Constellation-X, and the sub-millimeter array SCUBA II. Large sensor arrays also provide increased collection area for applications in materials analysis.

However, the undegraded measurement of signals from thousands of sensors and the transport of these signals from below $1 \mathrm{~K}$ to room temperature pose significant technical challenges. In order to realize the next-generation instruments mentioned above, techniques to minimize the number of interconnects and read-out amplifiers are required. Two proposed methods for reading out large arrays of TES sensors are time and frequency-domain multiplexing ${ }^{5,6,7}$. While timedomain multiplexing has been successfully demonstrated ${ }^{8}$, frequency-domain multiplexers that combine all of the features needed for a practical system are still under development.

In this letter, we present a frequency-domain multiplexing measurement in which two sensors are measured with a single amplifier. As shown in Fig. 1, each TES is biased at an identifying frequency and operated in a series resonant circuit to suppress out-of-band noise. The current through each sensor couples inductively to a superconducting loop and the summed 
signal in the loop is measured with a Superconducting QUantum Interference Device (SQUID). We demonstrate that the energy resolution of the sensors is unaffected by multiplexing and that cross-talk between the sensors is negligible. In addition, we discuss the optimal selection of circuit parameters and the limits on the number of sensors that can be measured in this manner.

The calorimeters used in this study are designed to analyze radioactive materials via high-resolution measurements of gamma-rays near $100 \mathrm{keV}$. The sensors consist of a superconducting Sn absorber ( $1 \mathrm{~mm}$ x $1 \mathrm{~mm}$ x $250 \mu \mathrm{m}$ ) glued to a Mo/Cu TES thermometer (500 $\mu \mathrm{m} \times 500 \mu \mathrm{m} \times 0.2 \mu \mathrm{m})$. The critical temperature of the devices is near $120 \mathrm{mK}$ and their normal resistance $\mathrm{R}_{\mathrm{n}}$ is about $0.35 \Omega$. Further details can be found elsewhere ${ }^{9}$.

When a sensor absorbs a photon, the temperature and resistance of the TES increase and the current through the TES decreases. In our devices, the diffusion of energy from the absorber to the thermometer takes approximately $100 \mu$ s and determines the rise time of the current pulse caused by a photon. The fall time $\tau_{\mathrm{f}}$ of the pulse depends on the strength of the electrothermal feedback in the device and the rate at which energy exits the absorber. In our devices, the shortest fall-times were approximately $1 \mathrm{~ms}$. The $3 \mathrm{~dB}$ point of the signal is given by $1 / 2 \pi \tau_{\mathrm{f}}=$ $160 \mathrm{~Hz}$ and the maximum signal bandwidth $v_{\mathrm{bw}}$, the point where the signal-to-noise ratio becomes unity, is $20 \mathrm{kHz}$.

Traditionally TES sensors have been operated with a static voltage bias provided by a current source and a small shunt resistor connected in parallel with the sensor. For frequencydomain multiplexing, the static voltage bias is eliminated and an alternating current with frequency $v_{\text {car }}$ is applied to each sensor and shunt resistor. As shown in Fig. 2, heating of the sensor by a photon increases the device resistance and decreases the amplitude of the alternating current flowing through the device. In the frequency domain, this transient amplitude 
modulation resides in sidebands of width $v_{\mathrm{bw}}$ above and below the carrier frequency $v_{\mathrm{car}}$.

Several authors have demonstrated operation of a single device under oscillating bias ${ }^{10,11,12}$.

For the circuit shown in Fig. 1 to function properly, it is necessary to correctly choose the bias frequencies, the inductances and capacitances of the sensor circuits, and the inductances of the summing loop. Since the bias heats the device above the bath temperature, the minimum bias frequency must be high enough that the temperature oscillations within the device caused by the bias do not affect device performance. We chose sinusoidal biases with frequencies $v_{1}=124$ $\mathrm{kHz}$ and $v_{2}=154 \mathrm{kHz}$ for devices \#1 and \#2, respectively. The corresponding bias periods are more than ten times shorter than the pulse rise times so the measured pulse heights are independent of the phase of the bias relative to the signal pulse. The spacing between the carrier frequencies was chosen to be approximately $2 v_{\mathrm{bw}}$ so that the signals from the devices do not overlap.

Because TES devices are resistive, they are a source of Johnson noise at all frequencies and it is necessary to operate each sensor in a series resonant filter to limit its noise contribution to the other sensors. The half-width of the filter resonance $\Delta \omega=\mathrm{R} / 2 \mathrm{~L}$ must be chosen to contain the signal bandwidth. For device \#1 (\#2) the circuit inductance was chosen to be $3.0(1.9) \mu \mathrm{H}$ and at $50 \% \mathrm{R}_{\mathrm{n}}$ the half-width of the filter was $4.9(7.7) \mathrm{kHz}$. The circuit capacitance is now determined by the choice of resonance frequency $\omega_{0}$, the circuit inductance, and the relation $\mathrm{C}=$ $1 / \mathrm{L}_{\mathrm{o}}^{2}$

The circuit used is shown in Fig. 1. Each sensor is coupled to a superconducting summing loop which contains eight transformers in series with a feedback transformer and the input coil of a 100-SQUID series array (Hypres Model 30). Only two of the sensor transformers are used in our experiment. The voltage output from the SQUID is amplified, integrated, and fed 
back via a resistance $\mathrm{R}_{\mathrm{fb}}$ to flux-lock the summing loop. The sensor and feedback transformers each contribute $0.19 \mu \mathrm{H}$ and the input inductance of the SQUID is $0.5 \mu \mathrm{H}$, so the total summing loop inductance is $2.2 \mu \mathrm{H}^{6}$. The measured step-down ratio of sensor current to loop current is 14. Nonetheless, the transformed noise current from the sensors overrides the input noise current of the SQUID.

The spectral density of the SQUID output is shown in Fig. 3 for several operating states. Device \#1 is biased at $20 \% \mathrm{R}_{\mathrm{n}}$ and data are shown when the device is quiescent and when it has absorbed a photon. It can be seen that the signal due to the photon spans less than half the separation between the carrier frequencies, as expected. Device \#2 is shown in several operating states; first, the bias is off, and then the bias is increased to yield operating resistances of $20 \%$ and $50 \% \mathrm{R}_{\mathrm{n}}$. As the resistance is increased from $20 \%$ to $50 \%$ of $\mathrm{R}_{\mathrm{n}}$, the device noise decreases due to the expected decrease in the phonon and Johnson noise. Noise from the SQUID dominates the unbiased case.

In order to measure gamma-ray pulses, the output of the SQUID amplifier was split at room temperature and demodulated using lock-in amplifiers (Perkin-Elmer 7280 and Stanford Research SR844) tuned to each carrier. The measured full-width half-maximum energy resolutions of devices \#1 and \#2 in response to $59.54 \mathrm{keV}$ gamma-rays from a ${ }^{241} \mathrm{Am}$ source are summarized in Table 1. For each device, three cases are presented: the energy resolution when the other device is unbiased, the energy resolution when the other device is biased but not receiving pulses, and the energy resolution when the other device is biased and has received a pulse within $20 \mathrm{~ms}$ of the pulse in the device of interest. It can be seen that the resolution of each sensor is unaffected by the bias of the other, and also unaffected by the presence of coincident pulses. The entries in Table 1 are average results from between two and twelve spectra, each 
containing between 300 and 900 counts in the $59.54 \mathrm{keV}$ peak. Furthermore, we characterized the same sensors under static bias and our results with an oscillating bias are the same or slightly better. We note that the energy resolutions in Table 1 are very good for superconducting sensors operating in this energy range and approximately a factor of five better than can be obtained with semiconducting detectors.

The number of sensors that can be measured by a single amplifier is limited by the bandwidth and dynamic range of the amplifier. The bandwidth of flux-locked SQUID systems can be $1 \mathrm{MHz}$ or more and we have demonstrated that the carrier frequencies can be spaced by $30 \mathrm{kHz}$ beginning at $124 \mathrm{kHz}$. If we take $1 \mathrm{MHz}$ to be the maximum bias frequency then there is sufficient bandwidth to multiplex 30 of our sensors.

The output of SQUIDs is periodic in the flux quantum $\phi_{o}$ and the fraction of the signal flux that is left uncancelled by the feedback must lie in a smaller range $\phi r$ for the SQUID response to be linear. This constraint both limits the maximum number of sensors and motivates an efficient feedback system. It is useful to divide down the sensor signals in order to reduce the flux burden on the SQUID. For undegraded operation, the device noise $I_{N d e v}$ should dominate the SQUID noise $I N s q$ yielding the relation $I N d e v / D=\chi I N s q$ where $D$ is the reduction in the signal and $\chi$ is the target ratio of device-to-SQUID noise. The peak flux burden on the SQUID is then given by $\sqrt{2} N I \operatorname{dev} M /(D A L G)$ where $N$ is the number of sensors, $A_{L G}$ is the loop-gain of the feedback system, $I_{d e v}$ is the root-mean-square amplitude of the bias current for one sensor, and $M$ is the mutual inductance between the SQUID and its input coil. We thus obtain $N=A_{L G} I_{N d e v} \phi_{r} /\left(\sqrt{2} \chi I_{N s q} I_{d e v} M\right)$. For our devices, INdev $=60 \mathrm{pA} / \sqrt{\mathrm{Hz}}$ and $I_{d e v} \approx 10 \mu \mathrm{A}$. For a Hypres SQUID array, INsq $=3 p A / \sqrt{H z}$ and $M=\phi_{o} / 14 \mu \mathrm{A}$. With careful design, it is possible to build a feedback system with $A_{L G} \approx 100$ over the required frequency range. ${ }^{13}$ For 
$A_{L G}=100, \chi=3$, and $\phi r=0.1 \phi_{o}$, we find $D=7$ and $N=66$. Techniques to null the flux from quiescent sensors can produce further increases in $N$. A more detailed analysis of these issues has recently been presented. ${ }^{13}$

In conclusion, we have multiplexed two TES microcalorimeters in the frequency domain without degrading their resolution or introducing cross-talk. We estimate that the technique can be extended to 30 or more of our sensors. These results are an important first step towards large sensor arrays for applications in astronomy and materials analysis.

This work was performed under the auspices of the U. S. DOE by the University of California, Lawrence Livermore National Laboratory under contract No. W-7405-ENG-48. The Berkeley group is supported by the Director, Office of Energy Research, Office of Basic Energy Science, Materials Sciences Division of the U. S. DOE under contract number DE-AC0376SF00098, and by NSF Grant AST-00-96933 and NASA Grant No. NAG5-11422. H.S. is supported by the Director, Office of Science, Office of High Energy and Nuclear Physics, of the U.S. DOE under Contract No. DE-AC03-76SF00098. 


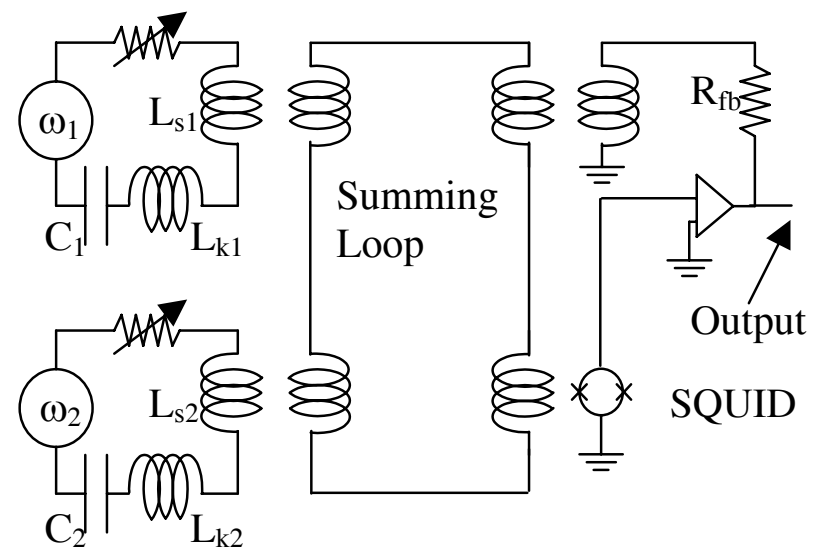

Fig. 1. Circuit schematic. Sensors are shown as variable resistors. Each TES is biased at an identifying frequency and operated in a series resonant circuit. The current in each TES circuit is inductively coupled by $\mathrm{L}_{\mathrm{s}}$ to the summing loop, which is inductively coupled to a SQUID amplifier. The current in the summing loop is reduced by feeding back the output of the SQUID. The summing loop is fabricated with input ports for eight sensors but only two are shown in the schematic. 


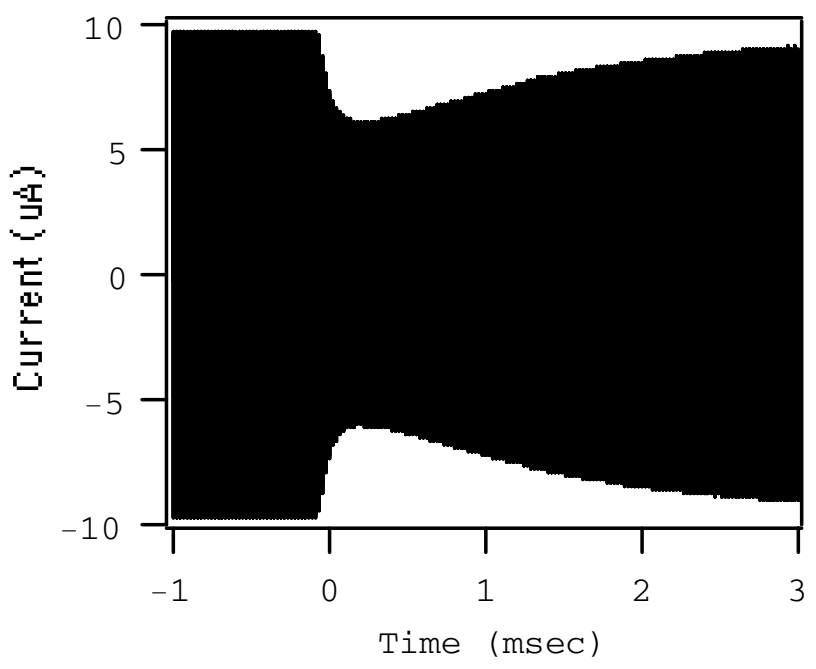

Fig. 2. Measured response of a single device under oscillating bias due to the absorption of a photon. The reduction in the amplitude of the carrier is proportional to the photon energy. 


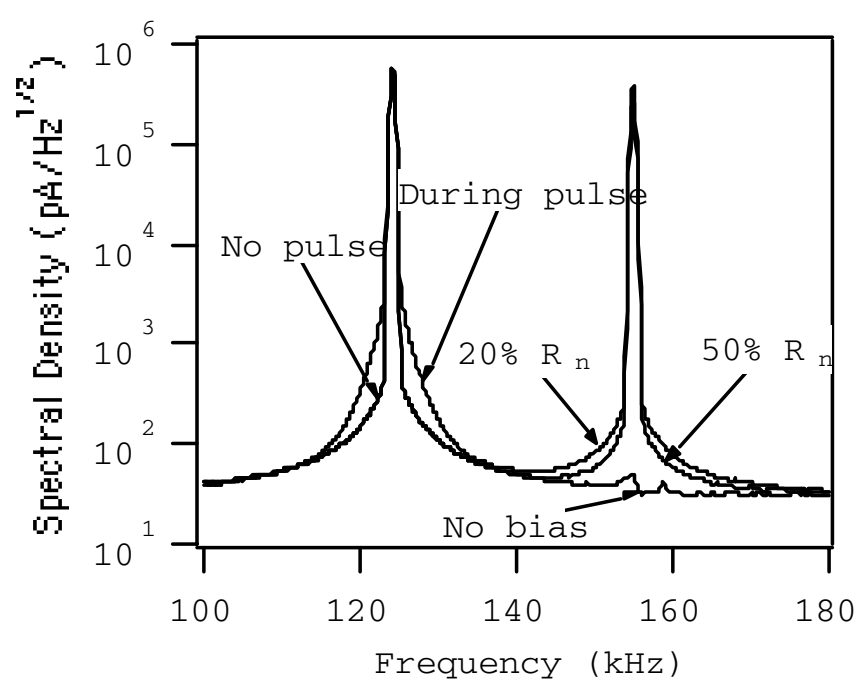

Fig. 3. Measured spectral density of two sensors under bias. Device $\# 1$ is held at $20 \% \mathrm{R}_{\mathrm{n}}$ and traces are shown with and without an absorbed photon. The bias of device \#2 is initially off and then adjusted so that the device is at $20 \%$ and then $50 \%$ of $\mathrm{R}_{\mathrm{n}}$.

\begin{tabular}{|l|l|l|l|} 
device & only recipient & both & both biased, \\
\hline
\end{tabular}




\begin{tabular}{|c|c|c|c|}
\hline & of bias & biased & coincident pulses \\
\hline \hline$\# 1$ & $65 \mathrm{eV}$ & $63 \mathrm{eV}$ & $64 \mathrm{eV}$ \\
\hline$\# 2$ & $62 \mathrm{eV}$ & $65 \mathrm{eV}$ & $63 \mathrm{eV}$ \\
\hline
\end{tabular}

Table 1. Measured energy resolutions. 


\section{List of References:}

1. D. T. Chow, A. Loshak, M. L. van den Berg, M. Frank, T. W. Barbee Jr., and S. E. Labov, Proc. SPIE 4141, 67 (2000).

2. K. D. Irwin, G. C. Hilton, J. M. Martinis, S. Deiker, N. Bergren, S. W. Nam, D. A. Rudman, and D. A. Wollman, Nucl. Instrum. Methods Phys. Res. A 444, 184 (2000).

3. B. Cabrera, R. M. Clarke, P. Colling, A. J. Miller, S. Nam, and R. W. Romani, Appl. Phys. Lett. 73, 735 (1998).

4. J. M. Gildemeister, Adrian T. Lee, and P. L. Richards, Appl. Phys. Lett. 74, 868 (1999).

5. J. A. Chervenak, K. D. Irwin, E. N. Grossman, J. M. Martinis, C. D. Reintsema, and M. E. Huber, Appl. Phys. Lett. 74, 4043 (1999).

6. Jongsoo Yoon, John Clarke, J. M. Gildemeister, Adrian T. Lee, M. J. Myers, P. L. Richards, and J. T. Skidmore, Appl. Phys. Lett. 78, 371 (2001).

7. T. Miyazaki, Ph.D. Thesis, University of Tokyo (2001).

8. D. J. Benford, T. A. Ames, J. A. Chervenak, E. N. Grossman, K. D. Irwin, S. A. Khan, B. Maffei, S. H. Moseley, F. Pajot, T. G. Phillips, J.-C. Renault, C. D. Reintsema, C. Rioux, R. A. Shafer, J. G. Staguhn, C. Vastel, and G. M. Voellmer, AIP Conf. Proc. 605, 589 (2002).

9. M. L. van den Berg, D. T. Chow, A. Loshak, M. F. Cunningham, T. W. Barbee Jr., M. Frank, and S. E. Labov, Proc. SPIE 4140, 436 (2000). 


\section{List of References (cont.):}

10. J. Yoon, J. Clarke, J. K. Gildemeister, A.T. Lee, and P. L. Richards, IEEE Transactions on Applied Superconductivity 11, 562 (2001).

11. M. F. Cunningham, J. N. Ullom, T. Miyazaki, O. Drury, A. Loshak, M. L. van den Berg, and S. E. Labov, AIP Conf. Proc. 605, 317 (2002).

12. J. van der Kuur, P.A .J. de Korte, H. F. C. Hoevers, M. Kiviranta, and H. Seppa, preprint submitted to Appl. Phys. Lett.

13. H. Spieler, LBNL-49993, to be published in the Proc. of the Far IR, sub-mm and mm Detector Technology Workshop, Monterey, CA, Apr. 1-3, 2002. 